Energy resol ved soft $x$ - $r$ ay i magi ng using a charge coupl ed devi ce camer a for I ong pul se di scharges in the Large Hel i cal Devi ce

\begin{tabular}{|l|l|}
\hline $\begin{array}{l}\text { j our nal or } \\
\text { publ i cat } i \text { on } \mathrm{t} \text { i t l e }\end{array}$ & Revi ew of Sci ent i f i c I nst r ument s \\
\hline vol une & Vol . 79 \\
\hline page r ange & 10E929-1 - 10E929- 4 \\
\hline year & 2008 $10-01$ \\
\hline URL & ht t p: //hdl . handl e. net /10655/3812 \\
\hline
\end{tabular}




\title{
Energy resolved soft x-ray imaging using a charge coupled device camera for long pulse discharges in the Large Helical Device ${ }^{a)}$
}

\author{
C. Suzuki, ${ }^{1}$ K. Ida, ${ }^{1}$ T. Kobuchi, ${ }^{2}$ and M. Yoshinuma ${ }^{1}$ \\ ${ }^{1}$ National Institute for Fusion Science, 322-6 Oroshi-cho, Toki 509-5292, Japan \\ ${ }^{2}$ Tohoku University, 6-6 Aoba, Aramaki, Aoba-ku, Sendai 980-8579, Japan
}

(Presented 13 May 2008; received 9 May 2008; accepted 30 May 2008; published online 31 October 2008)

\begin{abstract}
Energy resolved soft $\mathrm{x}$-ray imaging system using a charge coupled device camera and a multifilter disk has recently been installed to the Large Helical Device for the measurements in the long pulse discharges. Eight images with different cutoff energies are measured sequentially during a single discharge by rotating a filter disk mounting eight beryllium filters with different thicknesses. A tangential line-integrated profile for a specific photon energy range can be obtained by taking intensity difference between two images measured with a filter pair of adjacent thicknesses. The typical photon energy corresponding to each difference ranges from 1.9 to $4.8 \mathrm{keV}$ with the bandwidth of $2-3 \mathrm{keV}$. In the initial results, the difference in the line-integrated soft $\mathrm{x}$-ray profile by the energy range has been clearly observed. This diagnostic method can possibly be applied to the observation of the dependence of two dimensional soft x-ray profile on photon energy range especially if local non-Maxwellian component appears in electron energy distribution function.

(c) 2008 American Institute of Physics. [DOI: 10.1063/1.2953676]
\end{abstract}

\section{INTRODUCTION}

Charge coupled device (CCD) cameras sensitive directly to soft x-ray photons have recently been applied to soft x-ray diagnostics of magnetically confined high temperature plasmas. ${ }^{1-4}$ If the energy resolved imaging is accomplished by a certain technique, this diagnostic method can possibly be applied to the detection of the difference in two dimensional (2D) soft x-ray profile caused by local nonMaxwellian component of electron energy distribution function. In the previous studies, ${ }^{1,2}$ spatially resolved soft x-ray energy spectra were obtained directly by operating the camera in photon counting mode. However, it takes extremely long exposure time to get good spatial resolution enough for the detection of slight profile change in the photon counting mode.

Another possible way to achieve the energy resolved measurement is to place metallic filters with various thicknesses in front of the camera operated in the imaging mode. Though the energy resolution obtained by exchanging filters is lower than that in the photon counting mode, it still seems to be enough to observe changes in emissivity profiles for different energy ranges if the filter thickness can be changed variously and quickly during a stable discharge. However, it is often difficult to quickly exchange the filters during a single discharge since typical discharge duration is several seconds in most magnetically confined high temperature plasma experiments.

In the Large Helical Device (LHD) experiments, long pulse discharges under quasi-steady-state whose electron

\footnotetext{
a) Contributed paper, published as part of the Proceedings of the 17th Topical Conference on High-Temperature Plasma Diagnostics, Albuquerque, New Mexico, May 2008.
}

density and temperature are of the order of $10^{19} \mathrm{~m}^{-3}$ and $1 \mathrm{keV}$, respectively, can be routinely sustained for several minutes. ${ }^{5}$ The energy resolved soft $\mathrm{x}$-ray imaging by a CCD camera may be realized in such long pulse discharges by changing the filter thickness during one shot. In this study we have applied this method for the energy resolved soft x-ray imaging by a CCD camera in long pulse discharges of the LHD for the first time. We report mainly the details of diagnostic aspects and some examples of the preliminary results in this article.

\section{EXPERIMENTAL SETUP}

A schematic of the diagnostic system installed in a tangential viewport of the LHD is shown in Fig. 1. Though the present system is basically similar to the one installed previously, ${ }^{2}$ components including a camera have been partly modified or upgraded for better performance. The heart of the system is a soft x-ray CCD camera (Andor Technology, DO435-BV) equipped with a back illuminated CCD chip of frame transfer type. The image area is $13.3 \times 13.3 \mathrm{~mm}^{2}$ composed of $1024 \times 1024$ pixels, and the same size of masked pixels are prepared for storage for the frame transfer. Since the CCD chip is cooled to $-65^{\circ} \mathrm{C}$ to minimize dark charge by a thermoelectric cooler, the dark noise is reduced to an ignorable level against the readout noise. The CCD camera is controlled by an extension board installed in a personal computer, including a $1 \mathrm{MHz}$ analog-to-digital converter with a resolution of 16 bits. The full well depth and the readout noise are 85000 and 22 electrons/pixel, respectively. The overall signal-to-noise ratio (SNR) is dominated by photon shot noise even in the case of the thickest filter used. The total readout time is about $0.6 \mathrm{~s}$ for $2 \times 2$ pixel binning. 


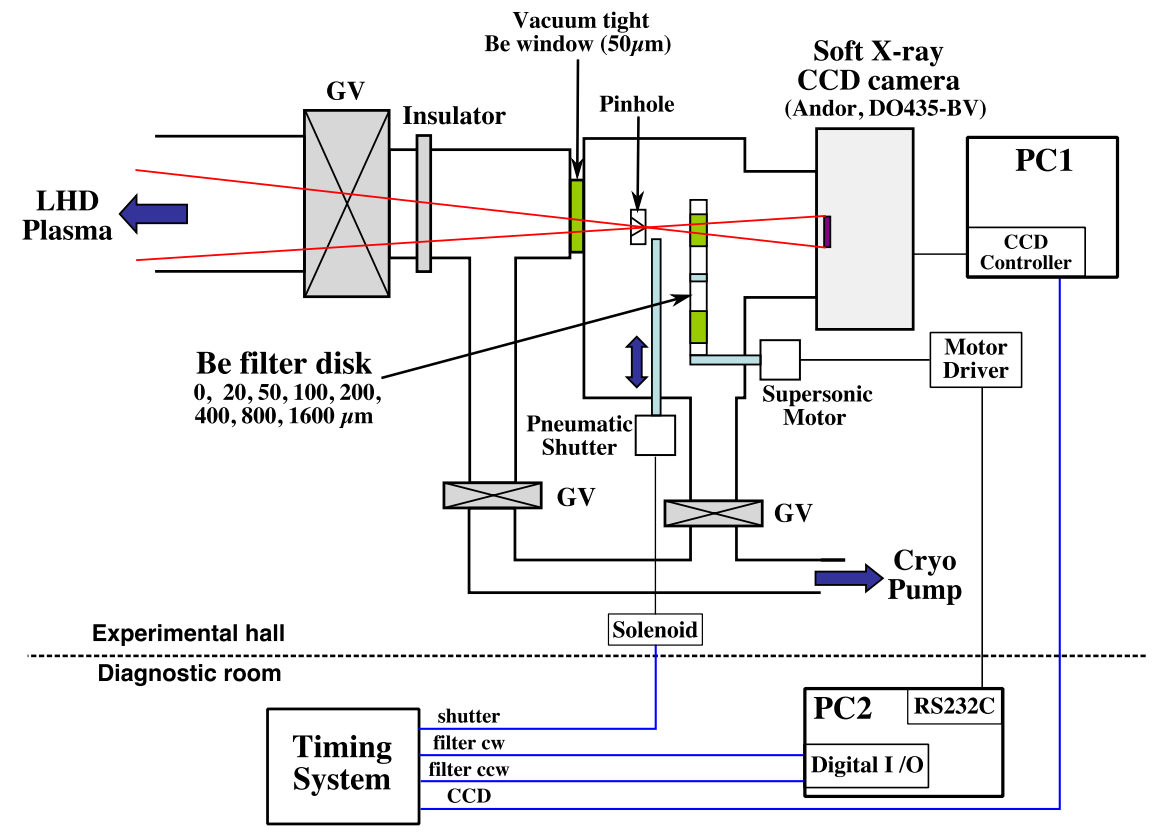

FIG. 1. (Color online) Schematic of the experimental setup.

A pneumatic mechanical shutter and a filter disk rotated remotely by a supersonic motor (Fukoku, USR-45W) have been employed to avoid the effect of the strong magnetic field around LHD. Eight slots are prepared for mounting filters on the filter disk at $45^{\circ}$ intervals. In the present study, we have employed beryllium (Be) filters whose thicknesses are selectable from 50, 70, 100, 150, 250, 450, 850, and $1650 \mu \mathrm{m}$, including a common $50 \mu \mathrm{m}$ Be window installed for the protection of the LHD vacuum. The diameter of the pinhole can also be chosen out of $0.05,0.1,0.2$, and $0.4 \mathrm{~mm}$.

Timings of the movement of the shutter, the CCD readout, and the rotation of the filter disk are controlled by four different trigger pulses generated by an existing timing system. The triggers for the shutter and the CCD camera are sent to a solenoid and the CCD controller board, respectively. The triggers for clockwise and counterclockwise rotations of the filter disk are fed into another personal computer, where commands are sent to a motor driver by a software via RS-232C interface. An example of the timing chart is shown in Fig. 2 in which the exposure time is $1 \mathrm{~s}$. The measurement begins with opening the shutter and reading out the initial dark charge of the CCD after waiting initial several seconds

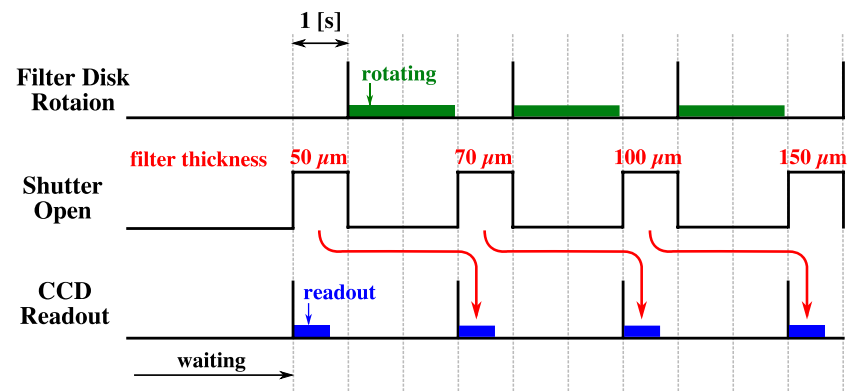

FIG. 2. (Color online) Timing chart of the filter disk rotation, the shutter open, and the CCD readout. of the discharge until the plasma reaches a quasi-steadystate. The rotation of the filter disk is triggered after the exposure to move to the next filter in the next $2 \mathrm{~s}$, in which the shutter is closed in order to avoid the light illumination onto the CCD during the movement. After the one-step rotation of the filter disk is finished, the next frame for the adjacent filter thickness begins with opening the shutter again, during which the image of the previous frame is read out simultaneously from the storage area of the frame transfer CCD. The frame rate in the present system is limited by the maximum rotation speed of the filter disk since it is slower than the readout time of the CCD. Namely, the minimum acquisition cycle time equals the readout time of the CCD plus $2 \mathrm{~s}$ since it takes at least $2 \mathrm{~s}$ to complete the one-step rotation.

The maximum charge stored in a CCD pixel is limited by the well depth of the shift register, which means that light illumination below the saturation level in full resolution mode may be saturated in binning operation. Since the exposure time is not variable in the present system, the dynamic range should be as large as possible. In addition, the SNR is dominated by shot noise in our system even in the full resolution mode. Therefore the measurement in the full resolution mode is likely to be an ideal choice. However, the full resolution mode causes some error in communication due probably to huge data size. Hence we have chosen 2 $\times 2$ pixel binning throughout this study.

\section{DIFFERENTIAL SENSITIVITY}

A tangential image for a specific photon energy range can be obtained by taking the difference between two signals measured with different filter thicknesses. The curve of overall differential sensitivity versus photon energy for the signal intensity difference can be calculated from the product of the filter transmission and the quantum efficiency of the CCD 


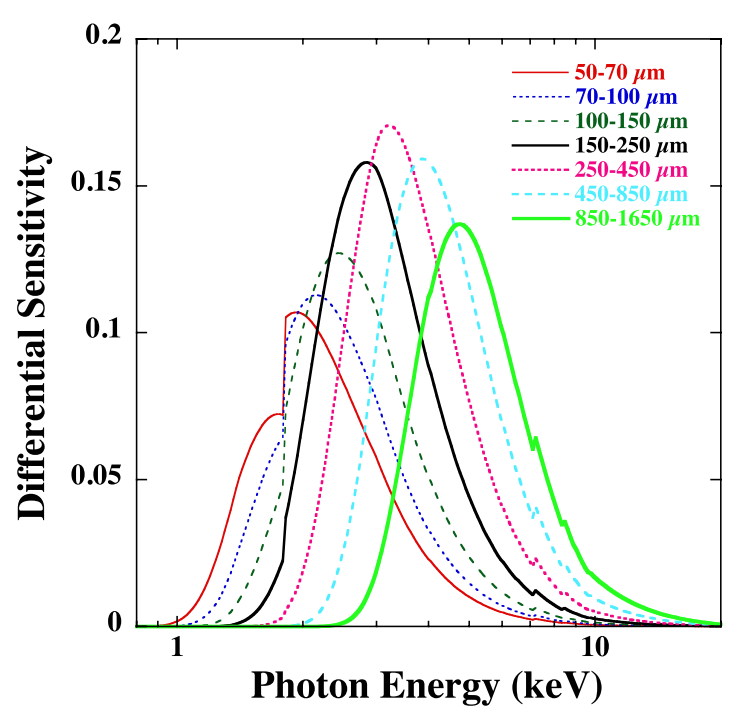

FIG. 3. (Color online) Differential sensitivity curves for the differences between adjacent Be filter thicknesses used in this study, including the CCD quantum efficiency.

chip provided by the manufacturer. Figure 3 shows differential sensitivity curves for the seven different combinations of the adjacent Be filter thicknesses used in this study. Typical photon energies observed for each difference correspond to the energies at the peaks of these curves, which range from 1.9 to $4.8 \mathrm{keV}$ with the bandwidth of $2-3 \mathrm{keV}$. A jump around $1.8 \mathrm{keV}$ is caused by an absorption edge of silicon in the CCD quantum efficiency curve. In this study, ultrahigh purity (99.8\%) Be foils are used. Though the effects of the impurities in the filter material are taken into account in Fig. 3 , the effects of the purity on the differential sensitivity are almost negligible. ${ }^{6}$

\section{RESULTS AND PERSPECTIVES}

Examples of the measured tangential images for the specific photon energy ranges are shown in Fig. 4, in which contours of the 2D images are drawn for the signal differences (a) between 50 and $70 \mu \mathrm{m}$ filter thicknesses, and (b) between 850 and $1650 \mu \mathrm{m}$, for the same discharge. These

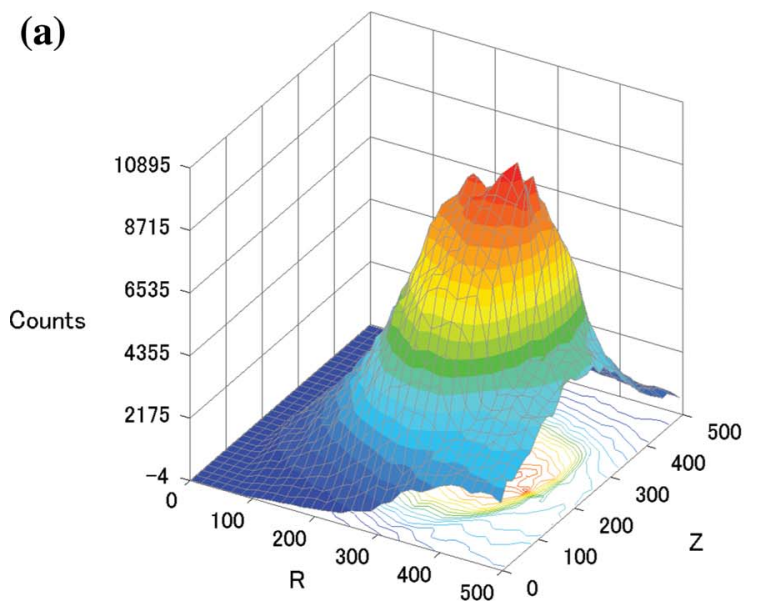

were measured in a long pulse discharge sustained by ion cyclotron resonance heating ${ }^{5}$ under quasi-steady-state. The acquisition cycle time and the exposure time were adjusted to 3 and $1 \mathrm{~s}$, respectively. The $2 \times 2$ hardware binning of the CCD pixels results in $512 \times 512$ superpixels in major radius $(R)$ and vertical $(Z)$ directions. In addition, $16 \times 16$ superpixels are averaged for smoothing (software binning) when the contours are drawn.

It is apparently found that the profile of Fig. 4(a) is rather broader than that of Fig. 4(b). According to Fig. 3, photon energies that mainly contribute to the signals are around 1.9 and $4.8 \mathrm{keV}$ for (a) and (b), respectively. Therefore the observed difference in the profile is basically reasonable since the profile of $4.8 \mathrm{keV}$ photons tends to be more peaked than that of the $1.9 \mathrm{keV}$ ones.

It is possible to estimate roughly line-averaged effective electron temperature by comparing the measured dependences of the line-integrated signal intensities on the energy range with the calculated ones for various electron temperatures, assuming continuum radiation and spatially uniform effective charge $\left(Z_{\text {eff }}\right){ }^{7}$ However, the $K_{\alpha}$ line radiations from metallic impurities would influence the observed signal intensity especially in the higher energy range, which is supposed from x-ray pulse height analyzer measurement. ${ }^{8}$ In addition, the uncertainty in overall filter thickness would result in relatively large error bars. ${ }^{6}$

We will develop another soft $\mathrm{x}$-ray CCD camera system mainly used for the photon counting mode in the near future as an alternative way to measure the effects of the soft $\mathrm{x}$-ray line spectra. Actual uncertainty of the filter thickness should be precisely measured for evaluating the error bars. In order to discuss spatial profiles in more detail, routines for the inversion of radial profiles based on a three dimensional equilibrium code is presently under preparation for the new system.

Finally, the measurement in a stable long pulse discharge by electron cyclotron resonance heating (ECRH) will be planned to study the effect of local non-Maxwellian component on the energy resolved soft x-ray profiles because the effect would be larger in the ECRH discharges. We have already estimated quantitatively the signal intensity ratio be-

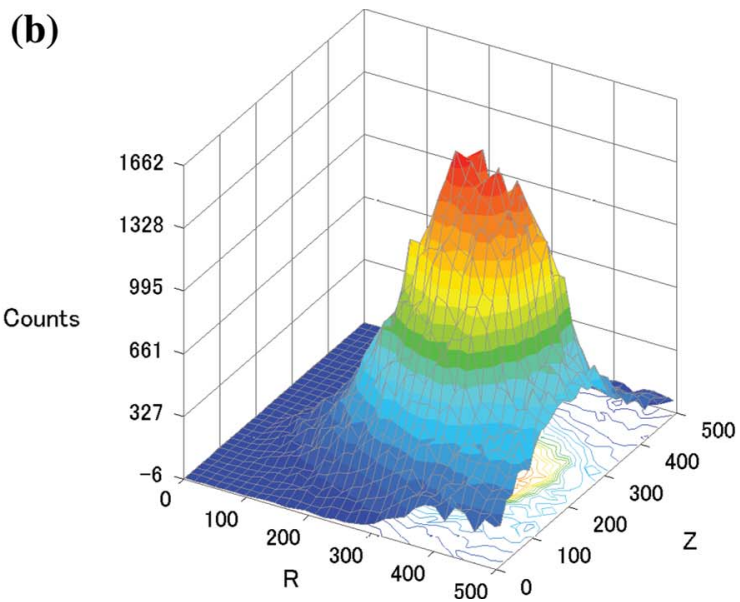

FIG. 4. (Color online) Contours of the 2D images obtained from the signal differences (a) between 50 and $70 \mu \mathrm{m}$ filter thicknesses, and (b) between 850 and $1650 \mu \mathrm{m}$, which correspond to the photon energy ranges around 1.9 and $4.8 \mathrm{keV}$, respectively. 
tween the highest and lowest energy ranges in this study, which is very sensitive to the change in the electron temperature. ${ }^{7}$ Therefore we believe that the distortion of the profile due to several percentage of the high energy component of electron energy distribution function would be easily observed in the measured images.

\section{ACKNOWLEDGMENTS}

This work has been partly supported by a Grant-in-Aid for Scientific Research from MEXT. This work was carried out under budget code NIFS07ULBB510. The authors acknowledge the LHD experimental group for their assistance.

${ }^{1}$ Y. Liang, K. Ida, S. Kado, I. Nomura, and T. Minami, Rev. Sci. Instrum. 71, 3711 (2000).

${ }^{2}$ Y. Liang, K. Ida, S. Kado, T. Minami, S. Okamura, I. Nomura, K. Y. Watanabe, H. Yamada, CHS Group, and LHD Group, Rev. Sci. Instrum. 72, 717 (2001)

${ }^{3}$ Y. Liang, K. Ida, S. Kado, K. Y. Watanabe, S. Sakakibara, M. Yokoyama, H. Yamada, A. Komori, K. Narihara, K. Tanaka, T. Tokuzawa, Y.
Nagayama, Y. Nakamura, N. Ohyabu, K. Kawahata, S. Sudo, and LHD Experimental Group, Plasma Phys. Controlled Fusion 44, 1383 (2002).

${ }^{4}$ T. Kobuchi, K. Ida, H. Yamada, M. Yokoyama, K. Y. Watanabe, S. Sakakibara, M. Yoshinuma, and the LHD Experimental Group, Plasma Phys. Controlled Fusion 48, 789 (2006).

${ }^{5}$ R. Kumazawa, T. Mutoh, K. Saito, T. Seki, Y. Nakamura, S. Kubo, T. Shimozuma, Y. Yoshimura, H. Igami, K. Ohkubo, Y. Takeiri, Y. Oka, K. Tsumori, M. Osakabe, K. Ikeda, K. Nagaoka, O. Kaneko, J. Miyazawa, S. Morita, K. Narihara, M. Shoji, S. Masuzaki, M. Kobayashi, H. Ogawa, M. Goto, T. Morisaki, B. J. Peterson, K. Sato, T. Tokuzawa, N. Ashikawa, K. Nishimura, H. Funaba, H. Chikaraishi, T. Watari, T. Watanabe, M. Sakamoto, M. Ichimura, Y. Takase, T. Notake, N. Takeuchi, Y. Torii, F. Shimpo, G. Nomura, C. Takahashi, M. Yokota, A. Kato, Y. Zhao, J. G. Kwak, J. S. Yoon, H. Yamada, K. Kawahata, N. Ohyabu, K. Ida, Y. Nagayama, N. Noda, A. Komori, S. Sudo, O. Motojima, and LHD Experimental Group, Nucl. Fusion 46, S13 (2006).

${ }^{6}$ C. Suzuki, K. Ida, T. Kobuchi, M. Yoshinuma, and LHD Experimental Group, Proceedings of the 17th ITC/ISHW Conference, Toki, Japan, 2007, P2-090.

${ }^{7}$ C. Suzuki, K. Ida, T. Kobuchi, M. Yoshinuma, and LHD Experimental Group, Proceedings of the 34th EPS Conference on Plasma Physics, Warsaw, Poland (EPS, Mulhouse, 2007), ECA Vol. 31F, P2.139.

${ }^{8}$ S. Muto, S. Morita, and LHD Experimental Group, Rev. Sci. Instrum. 72, $1206(2001)$ 